\title{
Complications and safety of vagus nerve stimulation: 25 years of experience at a single center
}

\author{
David Révész, MD,1,2 Bertil Rydenhag, MD, PhD, ${ }^{1,2}$ and Elinor Ben-Menachem, MD, $\mathrm{PhD}^{1,3}$ \\ ${ }^{1}$ Institute of Neuroscience and Physiology, Department of Clinical Neuroscience and Rehabilitation, The Sahlgrenska Academy \\ at the University of Gothenburg; and Departments of ${ }^{2}$ Neurosurgery and ${ }^{3}$ Neurology, Sahlgrenska University Hospital, \\ Gothenburg, Sweden
}

\begin{abstract}
OBJECTIVE The goal of this paper was to investigate surgical and hardware complications in a longitudinal retrospective study.

METHODS The authors of this registry study analyzed the surgical and hardware complications in 247 patients who underwent the implantation of a vagus nerve stimulation (VNS) device between 1990 and 2014. The mean follow-up time was 12 years.
\end{abstract}

RESULTS In total, 497 procedures were performed for 247 primary VNS implantations. Complications related to surgery occurred in $8.6 \%$ of all implantation procedures that were performed. The respective rate for hardware complications was $3.7 \%$. Surgical complications included postoperative hematoma in $1.9 \%$, infection in $2.6 \%$, vocal cord palsy in $1.4 \%$, lower facial weakness in $0.2 \%$, pain and sensory-related complications in $1.4 \%$, aseptic reaction in $0.2 \%$, cable discomfort in $0.2 \%$, surgical cable break in $0.2 \%$, oversized stimulator pocket in $0.2 \%$, and battery displacement in $0.2 \%$ of patients. Hardware-related complications included lead fracture/malfunction in 3.0\%, spontaneous VNS turn-on in $0.2 \%$, and lead disconnection in $0.2 \%$ of patients.

CONCLUSIONS VNS implantation is a relatively safe procedure, but it still involves certain risks. The most common complications are postoperative hematoma, infection, and vocal cord palsy. Although their occurrence rates are rather low at about $2 \%$, these complications may cause major suffering and even be life threatening. To reduce complications, it is important to have a long-term perspective. The 25 years of follow-up of this study is of great strength considering that VNS can be a life-long treatment for many patients. Thus, it is important to include repeated surgeries such as battery and lead replacements, given that complications also may occur with these surgeries.

http://thejns.org/doi/abs/10.3171/2016.1.PEDS15534

KEY WORDS epilepsy; treatment; neurosurgery; vagus nerve stimulation; VNS; implants; complications

$\mathrm{V}$ AGUS nerve stimulation (VNS) is a therapy that has been available for the treatment of refractory epilepsy since 1994 in Europe and 1997 in the US. VNS was also approved for treating depression in 2001 in Europe and 2005 in the US. Our department was one of the very first to begin VNS implantation in 1990 when the first trials (EO3) were initiated ${ }^{8,32}$ and has continued to perform implantations ever since, with a relatively stable number of operations every year. Despite over 20 years of availability, discussion of safety and efficacy is still ongoing. The new evidence-based guidelines from the American Academy of Neurology (AAN) (2013) that analyzed all VNS studies until 2012 emphasized the need for further safety information. The major safety questions from the evidence-based analysis that still need to be clarified are if new safety concerns have emerged over time for sudden unexpected death in epilepsy (SUDEP), cardiac arrhythmias during and after implantation, and sleep apnea. ${ }^{26} \mathrm{~A}$ recent review presents safety data from clinical trials and databases, ${ }^{9}$ and results concerning SUDEP and patients with VNS from a single center were also reported recently. ${ }^{16}$ The long-term follow-up is limited in most studies; however, Kahlow and Olivecrona ${ }^{21}$ presented data over a 17-year period between 1994 and 2010.

Safety concerns must be seen in long-term perspectives. Many patients receive their VNS when they are young and

ABBREVIATIONS AAN = American Academy of Neurology; EEG = electroencephalography; IQR = interquartile range; SUDEP = sudden unexplained death in epilepsy;

VNS = vagus nerve stimulation.

SUBMITTED August 30, 2015. ACCEPTED January 13, 2016.

INCLUDE WHEN CITING Published online March 25, 2016; DOI: 10.3171/2016.1.PEDS15534. 
are expected to use it for many years. At our center, all VNS patients have been recorded in a local registry since the start of VNS implantation. Thus, we have the possibility of analyzing long-term safety and efficacy aspects up to 25 years after implantation. Unique to our patient population are the very old patients who received VNS during a pilot study on Alzheimer's disease and clinical symptoms. ${ }^{25}$

\section{Methods}

All patients in this case series suffered from refractory epilepsy of different etiologies and syndromes. Patients whose epilepsy is refractory, irrespective of syndrome, were evaluated by the multidisciplinary epilepsy team at Sahlgrenska University Hospital, Gothenburg, Sweden, as potential suitable candidates. In the preimplantation evaluation, all patients were evaluated with respect to possible resective or palliative epilepsy surgery, i.e., callosotomy. This evaluation includes history, semiology, imaging (MRI since 1994), electroencephalography (EEG), and, for some patients, video-EEG monitoring. In total, 247 patients with epilepsy (age range 4-71 years; mean 29.5 years) were implanted with VNS between 1990 and 2014 . They had either not been considered as candidates for epilepsy surgery or had undergone failed epilepsy surgery.

For the subgroup analyses, patients were divided into adults and children, male and female, and procedures performed between 1990 and 1999 or 2000 and 2014.

All patients underwent the implantation of a VNS device (Cyberonics Inc. $[\mathrm{n}=242]$ or BioControl Medical [n =5]), mainly for drug-resistant epilepsy, at our department between January 1990 and December 2014. Apart from primary surgery, all replacements due to battery failure were analyzed to determine the occurrence of surgical and hardware complications.

Standard preoperative chlorhexidine scrubbing and blood work were performed on all patients. If body temperature and C-reactive protein were considered pathologically elevated, the procedure was postponed. After the administration of antibiotics (either $1.5 \mathrm{~g}$ cefuroxime or $2 \mathrm{~g}$ cloxacillin), the left vagus nerve was exposed via a transverse incision at a midcervical level. Initially the incision was longitudinal, but this technique was abandoned in 2003. As a standard procedure, a 2-mm lead was placed around the vagus nerve, tunneled, and connected to the stimulator. Three-millimeter leads were used only during lead revision with prominent nerve fibrosis. The stimulator was then placed in a subcutaneous pocket over the left major pectoral muscle, and the wounds were closed. All patients who underwent any kind of revision, replacement, or extraction of the VNS received prophylactic antibiotics preoperatively, as is the custom at our center. The patients were under continuous surveillance the first night after surgery and most went home the following day. There were no outpatient procedures. All patients, children and adults, underwent the same surgical procedure.

If no immediate complication occurred postoperatively, the patient was discharged the day after surgery. The patient revisited the hospital 1-2 weeks after surgery for initiation of the stimulator either by the epilepsy physician or epilepsy nurse. Patients were continuously evaluated at our clinic as well as the pediatric neurology department for outcomes and complications every 3 months for the 1st year, followed by 6 -month or yearly assessments. A few patients moved from the Gothenburg region and were lost to follow-up $(\mathrm{n}=3)$.

The generator was usually started after 2 weeks. The rationale for the time delay was the concept of nerve swelling following surgery that was later confirmed in the study by El Tahry et al. ${ }^{13}$ The device was started at 0.25 $\mathrm{mA}, 30 \mathrm{~Hz}$, and $500-\mu \mathrm{sec}$ pulse width for 30 seconds on and 5 minutes off. This was later changed if patients complained of vocal side effects, whereby the frequency was reduced to $20 \mathrm{~Hz}$ and the pulse width to $250 \mu \mathrm{sec}$. To improve efficacy, the settings were adjusted in some patients to 30 seconds on and 1.8 or 3 minutes off. Rapid cycling was tried in some patients ( 7 seconds on and 21 seconds off), but because of the shortened battery life and lack of improved efficacy, all patients were reverted back to 30 seconds on and 1.8-5 minutes off. Invariably, the magnet settings were 1 notch more than the regular stimulation.

\section{Statistical Analysis}

The continuous variables are presented both as the mean with standard deviation (SD) and as the median with interquartile range (IQR). The chi-square test was used for the comparison of proportions between groups. A p value $<0.05$ was considered to be statistically significant. All statistical calculations were performed using SPSS version 22 (IBM Corp.).

\section{Results}

Between January 1990 and December 2014, 260 patients were implanted with a VNS device. Thirteen of these patients were implanted with VNS as part of study on Alzheimer disease (age range 58-81 years), ${ }^{25}$ and the remaining 247 patients were diagnosed with epilepsy. Fifty-five of the patients were children (age range 4-18 years). Previous resective surgery had been performed in 52 of these patients (29 children). Patient characteristics are shown in Table 1.

The median follow-up time was 11.8 years (IQR 6.917.7), and the mean follow-up time was 12.0 years (SD 6.5). Table 2 shows a summary of the 497 surgical procedures performed. The mean time to stimulator replacement in all models was 5.6 years (SD 2.1), and the median time was 5.1 years (IQR 4.1-6.9). In all patients, the stimulator replacement was performed in the same pocket as was previously used in the primary implantation. Table 3 shows time to replacement in different models.

In total, there were 37 surgical complications and 16 hardware complications in 47 different patients. Surgical complications occurred in $6.8 \%$ of the procedures performed in children and $9.1 \%$ in adults. The difference was not significant $(\mathrm{p}=0.67)$. No technical complications were reported in children. All complications are summarized in Table 4.

A total of 8 incidents of postoperative hematomas were reported. One child in the second cohort suffered from a postoperative hematoma. Seven of the hematomas occurred after primary implantation, including the hemato- 
TABLE 1. Patient demographics

\begin{tabular}{lccccc}
\hline \multicolumn{1}{c}{ Variable } & Total & Children & Adults & Female & Male \\
\hline No. of patients & 247 & 55 & 192 & 125 & 122 \\
\hline Mean age, yrs (SD) & $29.5(14.8)$ & $11.8(3.6)$ & $34.6(12.7)$ & $29.3(14.8)$ & $29.8(14.9)$ \\
\hline Median age, yrs (IQR) & $27(19-39)$ & $12(9-15)$ & $32(24-43)$ & $26(19-38)$ & $28.5(18-41)$ \\
\hline No. of males (\%) & $122(49.4)$ & $30(54.6)$ & $92(47.9)$ & & \\
\hline No. of adults (\%) & $192(77.7)$ & & & $100(80.0)$ & $92(75.4)$ \\
\hline
\end{tabular}

TABLE 2. Summary of surgical procedures

\begin{tabular}{lrrrrrrr}
\hline \multicolumn{1}{c}{ Surgical Procedures } & Total & Children & Adults & Female & Male & $1990-1999$ & $2000-2014$ \\
\hline VNS implantation & 247 & 55 & 192 & 125 & 122 & 89 \\
\hline Plain stimulator replacement in 1 session & 161 & 29 & 132 & 68 & 93 & 29 \\
\hline System replacement in 1 session (stimulator \& lead) & 8 & 2 & 6 & 3 & 5 & 2 & 132 \\
\hline Plain lead replacement in 1 session & 9 & 1 & 8 & 4 & 5 & 4 & 5 \\
\hline Reimplantation of stimulator & 4 & 1 & 3 & 2 & 2 & 0 \\
\hline Explantation of stimulator & 45 & 9 & 36 & 21 & 24 & 14 & 3 \\
\hline Lead exploration & 5 & 0 & 5 & 2 & 3 & 4 \\
\hline Wound revision & 5 & 0 & 5 & 2 & 3 & 1 \\
\hline Explantation of lead & 4 & 1 & 3 & 3 & 1 & 0 \\
\hline Intentional stimulator replacement discontinued & 1 & 0 & 1 & 0 & 1 & 0 \\
\hline Intentional lead replacement discontinued & 1 & 0 & 1 & 1 & 0 & 1 \\
\hline Reconnection of lead to stimulator & 2 & 0 & 2 & 0 & 2 & 0 \\
\hline Repositioning of lead & 1 & 0 & 1 & 1 & 0 & 1 \\
\hline Reposition of stimulator & 2 & 0 & 2 & 2 & 0 & 1 \\
\hline Removal of postoperative hematoma & 2 & 0 & 2 & 1 & 1 & 1 \\
\hline Total surgical procedures & 497 & 98 & 399 & 235 & 262 & 1 \\
\hline
\end{tabular}

ma in the child. Two adults needed immediate hematoma evacuation due to compression of the trachea and risk of desaturation. Neither of these patients suffered any damage related to the reoperations. The remaining patients who experienced a postoperative hematoma were treated conservatively. One patient had a postoperative hematoma that occurred after stimulator replacement. This was followed by a postoperative infection, resulting in explantation of the stimulator. To monitor any deterioration due do the hematoma, the patients stayed in the postoperative ward overnight and, if they were stable the day after, they were discharged; thus, all postoperative hematomas were considered a surgical complication.

Eleven incidents of infection were reported in 10 patients (4 children), where 1 adult patient was reinfected after stimulator reimplantation. Eight infections occurred after primary implantation and 2 after stimulator replacement. All patients suffering from infection received oral antibiotics as the first line of treatment. Two patients (1 child) suffered from superficial infection and responded to oral antibiotics. The remaining patients ( 3 children) were treated with surgical removal of either the stimulator only, or both the stimulator and lead. The surgical treatment regimens and time frames are presented in Table 5. Mean time from implantation to surgery due to infection, wound revision or explantation was 99 days (SD 68; median 86 days). The mean age of the patients with infection was 22.1 years (SD 12.8; median 20.5 years). The infection rate between 1990 and 1999 was $0.8 \%$ and $3.3 \%$ between 2000 and 2014. The difference was, however, not statistically significant, with a $\mathrm{p}$ value of 0.057 .

Postoperative vocal cord paralysis was seen in 5 patients (no children), 4 after primary implantation and 1 after lead revision, and lower facial weakness in 1 patient. One patient suffered from facial tingling after surgery. All of these patients recovered from their initial symptoms after 2-14 months.

Five patients (no children) suffered from postoperative pain in the implantation area. Three of these patients recovered spontaneously, 1 underwent removal of the stimulator, and 1 had a surgical repositioning of the stimulator. In 1 case the pocket size was reduced when the stimulator

TABLE 3. Stimulator durability

\begin{tabular}{lccc}
\hline Model & No. of Patients & Mean Yrs (SD) & Median Yrs (IQR) \\
\hline 100 & 32 & $3.7(1.4)$ & $3.7(2.8-4.5)$ \\
\hline 101 & 30 & $4.3(1.0)$ & $4.4(3.9-4.7)$ \\
\hline $102 R$ & 71 & $6.8(2.0)$ & $6.7(5.1-8.4)$ \\
\hline 102 & 15 & $6.5(1.5)$ & $6.7(5.1-8.4)$ \\
\hline
\end{tabular}


TABLE 4. Summary of complications

\begin{tabular}{|c|c|c|c|c|c|c|c|}
\hline Complications & Total & Children & Adults & Female & Male & 1990-1999 & 2000-2014 \\
\hline Surgical complications (total) & $37(8.6 \%)$ & $6(6.8 \%)$ & $31(9.1 \%)$ & $25(12.4 \%)$ & $12(5.3 \%)$ & $11(8.9 \%)$ & $28(9.2 \%)$ \\
\hline Hematoma & $8(1.9 \%)$ & $1(1.1 \%)$ & $7(2.1 \%)$ & $6(3.0 \%)$ & $2(0.9 \%)$ & $2(1.6 \%)$ & $6(2.0 \%)$ \\
\hline Post-primary implantation & $7\left(2.8 \%{ }^{*}\right)$ & $1\left(1.8 \%{ }^{*}\right)$ & $6(3.1 \% *)$ & $6\left(4.8 \%{ }^{*}\right)$ & $1\left(0.8 \%{ }^{*}\right)$ & $2(2.2 \%)$ & $5(3.2 \%)$ \\
\hline Post-stimulator replacement & $1(0.6 \% \dagger)$ & $0(0 \% \dagger)$ & $1(0.7 \% \dagger)$ & $0(0 \% \dagger)$ & $1(1.0 \% \dagger)$ & $0(0 \%)$ & $1(0.7 \%)$ \\
\hline Infections (all) & $11(2.6 \%)$ & $4(4.5 \%)$ & $7(2.1 \%)$ & $5(2.5 \%)$ & $6(2.6 \%)$ & $1(0.8 \%)$ & $10(3.3 \%)$ \\
\hline Post-primary implantation & $8\left(3.2 \%{ }^{*}\right)$ & $4\left(7.3 \%{ }^{*}\right)$ & $4\left(2.1 \%{ }^{*}\right)$ & $4\left(3.2 \%{ }^{*}\right)$ & $4\left(3.3 \%{ }^{*}\right)$ & $0(0 \%)$ & $8(5.1 \%)$ \\
\hline Post-stimulator replacement & $2(1.1 \% \dagger)$ & $0(0 \% \dagger)$ & $2(1.4 \% \dagger)$ & $0(0 \% \dagger)$ & $2(1.9 \% \dagger)$ & $1(2.9 \%)$ & $1(0.7 \%)$ \\
\hline Post-stimulator reimplantation & $1(\mathrm{NA})$ & 0 (NA) & $1(\mathrm{NA})$ & $1(\mathrm{NA})$ & $0(\mathrm{NA})$ & $0(0 \%)$ & $1(0.3 \%)$ \\
\hline Vocal cord palsy (all) & $6(1.4 \%)$ & $0(0 \%)$ & $6(1.8 \%)$ & $5(2.5 \%)$ & $1(0.4 \%)$ & $3(2.4 \%)$ & $3(1.0 \%)$ \\
\hline Post-primary implantation & $5(2.0 \% *)$ & $0\left(0 \%{ }^{*}\right)$ & $5\left(2.6 \%{ }^{*}\right)$ & $4\left(3.2 \%{ }^{*}\right)$ & $1\left(0.8 \%{ }^{*}\right)$ & $2(2.2 \%)$ & $3(1.9 \%)$ \\
\hline Post-lead explantation or replacement & $1(\mathrm{NA})$ & $0(N A)$ & $1(\mathrm{NA})$ & $1(\mathrm{NA})$ & $0(\mathrm{NA})$ & $1(\mathrm{NA})$ & $0(\mathrm{NA})$ \\
\hline Lower facial weakness & $1(0.2 \%)$ & $0(0 \%)$ & $1(0.3 \%)$ & $1(0.5 \%)$ & $0(0 \%)$ & $1(0.8 \%)$ & $0(0 \%)$ \\
\hline Pain \& sensory-related complications & $6(1.4 \%)$ & $1(1.1 \%)$ & $5(1.5 \%)$ & $4(2.0 \%)$ & $2(0.9 \%)$ & $2(1.6 \%)$ & $4(1.3 \%)$ \\
\hline Bradycardia & $0(0 \%)$ & $0(0 \%)$ & $0(0 \%)$ & $0(0 \%)$ & $0(0 \%)$ & $0(0 \%)$ & $0(0 \%)$ \\
\hline Puncture of jugular vein & $0(0 \%)$ & $0(0 \%)$ & $0(0 \%)$ & $0(0 \%)$ & $0(0 \%)$ & $0(0 \%)$ & $0(0 \%)$ \\
\hline Large cutaneous nerve cutoff & $0(0 \%)$ & $0(0 \%)$ & $0(0 \%)$ & $0(0 \%)$ & $0(0 \%)$ & $0(0 \%)$ & $0(0 \%)$ \\
\hline Aseptic reaction & $1(0.2 \%)$ & $0(0 \%)$ & $1(0.3 \%)$ & $0(0 \%)$ & $1(0.4 \%)$ & $0(0 \%)$ & $1(0.3 \%)$ \\
\hline Cable discomfort & $1(0.2 \%)$ & $0(0 \%)$ & $1(0.3 \%)$ & $1(0.5 \%)$ & $0(0 \%)$ & $1(0.8 \%)$ & $0(0 \%)$ \\
\hline Surgical cable break & $1(0.2 \%)$ & $0(0 \%)$ & $1(0.3 \%)$ & $1(0.5 \%)$ & $0(0 \%)$ & $1(0.8 \%)$ & $0(0 \%)$ \\
\hline Oversized stimulator pocket & $1(0.2 \%)$ & $0(0 \%)$ & $1(0.3 \%)$ & $1(0.5 \%)$ & $0(0 \%)$ & $0(0 \%)$ & $1(0.3 \%)$ \\
\hline Battery displacement & $1(0.2 \%)$ & $0(0 \%)$ & $1(0.3 \%)$ & $1(0.5 \%)$ & $0(0 \%)$ & $0(0 \%)$ & $1(0.3 \%)$ \\
\hline Technical complications (total) & $16(3.7 \%)$ & $0(0 \%)$ & $16(4.7 \%)$ & $5(2.5 \%)$ & $11(4.8 \%)$ & $9(7.3 \%)$ & $7(2.3 \%)$ \\
\hline Lead fracture/lead malfunction & $13(3.0 \%)$ & $0(0 \%)$ & $13(3.8 \%)$ & $4(2.0 \%)$ & $9(4.0 \%)$ & $8(6.5 \%)$ & $5(1.6 \%)$ \\
\hline Spontaneous VNS turn-on & $1(0.2 \%)$ & $0(0 \%)$ & $1(0.3 \%)$ & $1(0.5 \%)$ & $0(0 \%)$ & $0(0 \%)$ & $1(0.3 \%)$ \\
\hline Lead disconnection & $2(0.5 \%)$ & $0(0 \%)$ & $2(0.6 \%)$ & $0(0 \%)$ & $2(0.9 \%)$ & $1(0.8 \%)$ & $1(0.3 \%)$ \\
\hline
\end{tabular}

NA = not applicable.

* Percentage of all primary VNS implantations (247 primary VNS implantations were performed on 55 children, 192 adults, 125 female patients, 122 male patients, 89 in 1990-1999, and 158 in 2000-2014).

† Percentage of all plain stimulator replacements in 1 session, system replacements in 1 session (stimulator and lead), plain lead replacements in 1 session, and reimplantations of the stimulator (182 procedures were performed on 33 children, 149 adults, 77 female patients, 105 male patients, 35 in $1990-1999$, and 147 in 2000-2014).

was changed and 1 case underwent exploration because the stimulator had turned in its subcutaneous pocket.

One 29-year-old patient underwent reoperation 3 months after primary implantation with lead placement correction because of discomfort and tightness on the throat. One aseptic reaction with fluid collection around the stimulator was reported. Although cultures were taken, no infectious microorganism was found when explanting the device. This occurred 6.2 years after primary implantation.

Thirteen lead malfunctions in 12 patients (no children) were reported. Lead exploration to try to salvage the lead was required in 5 cases, 1 was successful and the remaining resulted in replacements. Table 6 summarizes the surgical revisions performed after lead malfunction. The rate of lead breaks between 1990 and 1999 was $7.3 \%$ and 2.3\% between 2000 and 2014. The difference was statistically significant $(\mathrm{p}=0.049)$.

In 2 adult patients, an immediate postoperative lead disconnection was suspected, resulting in an early surgical reconnection. This was probably due to unsuccessful con- nection between the lead and stimulator during primary surgery. It is unclear if the surgeon performed a proper lead test in these cases. In 1 patient, the stimulator was turned on immediately after surgery despite being set to off-mode in the operating room. Thirty stimulators were removed due to the lack of efficacy in combination with discomfort from the stimulator or patient desire.

\section{Discussion}

The aim of this long-term follow-up study of all patients implanted with VNS since 1990 is to describe the panorama of complications to VNS implantations since its introduction in clinical trials at a single center. The Phase III clinical trials for VNS began in 1990, and our center was one of the first to participate in Europe. Initially, 18 patients were included, and since then a total of 245 patients have been implanted, including the patients in the Alzheimer study ${ }^{36}$ and the 3 patients lost to follow-up. This case series is, to our knowledge, the first longitudinal series dealing with complications to vagus nerve stimulation that ranges up to 25 years. Most long-term studies have 
TABLE 5. Infections

\begin{tabular}{|c|c|c|}
\hline Patient Sex (age in yrs) & Reoperation No. & Surgical Procedures Performed \& Time Btwn Surgeries \\
\hline \multicolumn{3}{|c|}{ Infections needing surgical revision following primary implantation } \\
\hline Female (6) & 1st & Lead removal after 26 days \\
\hline Female (11) & $1 \mathrm{st}$ & Stimulator removal after 34 days \\
\hline Male (11) & $1 \mathrm{st}$ & Complete system removal after 106 days \\
\hline Male (24) & 1 st & Complete system removal after 155 days \\
\hline \multirow[t]{4}{*}{ Male (26) } & $1 \mathrm{st}$ & Wound revision after 37 days \\
\hline & 2nd & Wound revision after 44 days \\
\hline & $3 r d$ & Stimulator removal after 49 days \\
\hline & 4th & Lead removal after 309 days \\
\hline \multirow[t]{6}{*}{ Female (30) } & $1 \mathrm{st}$ & Stimulator removal after 181 days \\
\hline & 2nd & Reimplantation of stimulator after 168 days \\
\hline & $3 \mathrm{rd}$ & Stimulator removal \& subtotal lead removal due to infection after 50 days \\
\hline & 4th & Wound revision after 40 days \\
\hline & 5th & Wound revision after 10 days \\
\hline & 6th & Removal of remaining lead after 24 days \\
\hline \multicolumn{3}{|c|}{ Infections needing surgical revision following stimulator replacement } \\
\hline \multirow[t]{2}{*}{ Male (37) } & 1st & Wound revision after 186 days \\
\hline & 2nd & Stimulator removal after 145 days \\
\hline \multirow[t]{2}{*}{ Male (40) } & 1 st & Stimulator removal after 65 days \\
\hline & 2nd & Reimplantation of stimulator after 141 days \\
\hline
\end{tabular}

safety data ranging from 1 to 5 years. ${ }^{6,14,19,20,34,37-39}$ Considering that VNS can be a life-long treatment for many patients, it also involves repeated surgeries with multiple (up to 4) battery replacements that we account for in our series.

The AAN guidelines on VNS were recently published and emphasized the lack of information or gaps in knowledge concerning the side effects of VNS, especially in the pediatric population. ${ }^{15,30,43}$ Therefore, we deem it important to present our comprehensive long-term data to help answer some of the concerns. Since the start of VNS implantation in 1990, a local registry of all VNS patients has been maintained. We have registry data concerning the efficacy and tolerability as well as side effects and implantation data, including difficulties encountered with a median follow-up time of 12 years, that, to our knowledge, is twice as long as any other report. ${ }^{14,21}$

The overall complication rate was $12.4 \%$, including both surgical and hardware complications. Most previous studies present frequencies ranging from $2.5 \%$ to $12.5 \% .^{2,11,15,18,22,23,34,38,39}$ It is reasonable to believe that the complication frequency increases with a longer follow-up time, considering the repeated surgeries needed due to depleted batteries and hardware being worn out. Kahlow and Olivecrona ${ }^{21}$ recently presented a single-center longitudinal study of 143 patients with a mean follow-up time of 62 months. ${ }^{21}$ The surgical complication frequency was $16.8 \%$, including 3 cases of perioperative jugular vein puncture. In the study by Elliott et al. ${ }^{14}$ with a mean follow-up time of 4.1 years, 12 patients experienced some degree of permanent vagus nerve injury, and there was also 1 case of pneumothorax. These unusual complications have never occurred at our site during the 25 years of follow-up. Others have reported vocal cord paralysis in 1\%-5.6\% of patients. $7,17,21,23,32,34,37,38,41$ The surgical complication rate for all procedures was $8.6 \%$, and $1.4 \%$ of the patients experienced vocal cord paralysis and all recovered completely. In a recent study by Robinson and Winston, ${ }^{33}$ vocal cord paralysis occurred at almost twice the rate with leads that had a 2-mm inner diameter in comparison with 3-mm inner diameter in patients 18 years of age and older. In our series, all patients were initially implanted with 2-mm inner-diameter leads. Only patients who underwent lead revision received a $3-\mathrm{mm}$ inner-diameter lead if fibrosis around the nerve was apparent. Lower facial weakness is a rare complication and is probably caused by high surgical incisions. The only case that occurred in our series was after a longitudinal skin incision at the time of implantation. Horizontal skin incision probably reduces the risk of lower facial weakness.

None of our patients experienced asystole during or after the implantation. Arrhythmia, including asystole and bradycardia, is an important but rare complication that has been reported in the literature. ${ }^{3-5,35,40}$ Possible reasons for this phenomenon could be polarity reversal of the leads during implantation causing efferent instead of afferent stimulation, ${ }^{44}$ indirect stimulation of the cervical cardiac nerves, technical malfunction of the device, or accidental over-manipulation of the nerve since placing the spiral (Cyberonics) lead around the nerve can be difficult due to anatomical variations. The CardioFit cuff lead is subjectively easier to apply because of its design and requires less manipulation of the vagus nerve as a whole. ${ }^{10}$ On the other hand, only a handful of CardioFit systems have been 
TABLE 6. Lead malfunctions

\begin{tabular}{|c|c|c|c|c|}
\hline Patient Sex (age in yrs) & Reoperation No. & Surgical Procedures Performed \& Time Btwn Surgeries & Model & Lead Model \\
\hline \multicolumn{5}{|c|}{ Lead malfunctions following primary implantation } \\
\hline \multirow[t]{2}{*}{ Female (21) } & 1 st & Lead exploration after 200 days & 100 & 300 \\
\hline & 2nd & Complete system replacement in 1 session after 3.9 yrs & 100 & 300 \\
\hline \multirow[t]{2}{*}{ Male (45) } & $1 \mathrm{st}$ & Lead exploration after 1.9 yrs & 100 & 300 \\
\hline & 2nd & Plain lead replacement after 48 yrs & 100 & 300 \\
\hline \multirow[t]{2}{*}{ Female (59) } & $1 \mathrm{st}$ & Plain lead replacement after $3.7 \mathrm{yrs}$ & 100 & 300 \\
\hline & 2nd & Plain lead replacement after 132 days & 100 & 300 \\
\hline Male (63) & 1 st & Complete system replacement in 1 session after 3.4 yrs & 102 & 304 \\
\hline Male $(70)$ & 1 st & Plain lead replacement after 3.7 yrs & 100 & 300 \\
\hline \multicolumn{5}{|c|}{ Lead malfunctions following stimulator replacement } \\
\hline Male (19) & 1st & Complete system replacement in 1 session after 9.6 years & 101 & 300 \\
\hline \multirow[t]{2}{*}{ Female (21) } & $1 \mathrm{st}$ & Plain lead replacement after 85 days & 101 & 300 \\
\hline & 2nd & Plain lead replacement (at 29 yrs of age, after another ordinary replacement) & 101 & 300 \\
\hline Male (42) & 1 st & Plain lead replacement after 13 days & 101 & 300 \\
\hline Male (49) & $1 \mathrm{st}$ & Lead exploration after $1.6 \mathrm{yrs}$ & 100 & 300 \\
\hline Male (53) & $1 \mathrm{st}$ & Plain lead replacement after 112 days & 100 & 300 \\
\hline \multirow[t]{2}{*}{ Male (56) } & 1 st & Lead exploration w/in a year (date of previous surgery unknown) & 100 & 300 \\
\hline & 2nd & Complete system replacement in 1 session after 28 days & & \\
\hline Male (59) & 1 st & Plain lead replacement after 291 days & 101 & 300 \\
\hline
\end{tabular}

implanted thus far, and it is too early to draw any conclusions about the complications rates. One can speculate that less manipulation of the nerve would decrease the number of instances of postoperative vocal cord paralysis.

Not all lead fractures were visible on radiography, and the lead failures were mainly detected by diminished function, e.g., increased seizure frequency and increased impedance measurement leading to revision or replacement of the lead. Only a few were detected on radiography, and the etiology of high impedance is not entirely clear. Some have described lead failure in the absence of visible fractures as "microlesions" within the lead cable. 12,42 Others have presented significant scar tissue around the lead as a possible cause of high impedance. ${ }^{29}$ We report 13 cases $(3.0 \%)$ of lead malfunction, of which no children were involved. Previous studies report $0.5 \%$ to $20.8 \%$. $^{11,15}$, $20,21,24,27,34,37,38$ Of the 13 lead malfunctions, all but 1 was of the Cyberonics model 300, which suggests that this model is more susceptible to breakage. This product is no longer distributed by Cyberonics, and lead failure has decreased significantly since the introduction of newer models. Some of our patients still have the original leads that were implanted between 1990 and 1994. It is interesting that most of the leads now show high impedance on "device diagnostics." However, these patients feel the stimulation especially during the use of the magnet, and the initial reduction of seizure frequency in the early years has remained stable. Because of the difficulties of replacing a lead, we have continued to stimulate with the original lead as long as the patient does not experience an increase in seizure frequency and/or severity. Explantation of the lead can be a challenge because of fibrosis around the nerve, ${ }^{7}$ and replacing the Cyberonics lead can take several hours using microscopic dissection. Postoperative hoarseness after lead replacement occurred in 1 patient. We have no experience of lead change with the CardioFit, as the oldest implanted device is just 3 years old.

The absolute majority of hardware complications occurred in the early years of implantation. This is probably due to both the learning curve and development of the equipment, both the leads and stimulators. In the early years, the same surgeon made all implantations. Since then, an additional 5 surgeons have learned the implantation technique. Lead quality and stimulator lifetime have increased and the stimulator size has decreased. This has resulted in fewer lead revisions and longer periods between stimulator replacements. When it comes to surgical techniques, the only difference in standard protocol is that the neck incision was longitudinal originally and today all neck incisions are transverse, which is more cosmetically appealing. The preoperative protocol has remained unchanged since the start of implantation.

Implantation in children has been carried out since 1994. No complications exclusive to this population have been encountered, except for an infection rate that was nearly twice as high as that in adults $(4.5 \%)$, which is in line with other previous studies. ${ }^{1,26}$ We speculate that the cause of this is excessive wound manipulation, especially in children with intellectual disabilities, which is common in this patient group. Close postoperative monitoring of this patient group might resolve this problem. No hardware complications were reported in the pediatric population, probably due to the fact that only 4 children were implanted between 1990 and 1999. The overall rate of infection was $2.6 \%$, and this is in the lower range compared with most previous complication studies where the infection rates are reported as 3\%-8\%..$^{31,34,37,38}$ When looking at the infection rates after battery replacement it was 
only $1.1 \%$, suggesting that this is a fairly low-risk procedure compared with primary implantation. In contrast to the recommendations from Air et al. ${ }^{1}$ to treat all deep infections with intravenous broad-spectrum antibiotics as the first-line treatment, our patients received oral antibiotics and all but 2 patients with infection had to have their stimulator removed. The incidence of infection was higher between 2000 and 2014. Even if not statistically significant, the higher infection rate in the later period might be due to the involvement of several surgeons.

Thirteen elderly patients $(<81$ years old $)$ also underwent implantation as part of a study on Alzheimer disease. ${ }^{25}$ None of these patients had epilepsy. Apart from 1 case of recurrence paralysis that recovered over a few months, no other complications were observed. Postoperative recovery was unremarkable. The follow-up time was relatively short compared with the epilepsy patients. They all died within 5 years due to their underlying disorder and are therefore not included in the mortality analysis for epilepsy patients. This implies that the implantation of VNS is well tolerated by all age groups.

Battery life has been extended but still varies between 2.8 and 8.2 years depending on the settings and the battery itself, with the Cyberonics 102R having the longest battery life.

Twenty-five patients treated with VNS for epilepsy have died over the 25 years of implantation at our center. When analyzing the cause of death, 2 patients were found to be definite SUDEP deaths and 5 others were possible SUDEP deaths. However, the cause of death was not traceable in some cases due to the fact that the patients had been treated at different hospitals. Excess mortality in patients with epilepsy has long been recognized, ${ }^{28}$ but the question still remains if the death rate as a result of SUDEP is lower in the VNS-treated population than in similar groups of patients with refractory epilepsy not treated with VNS.

Analyzing registry data over long time periods can be difficult, especially as surgeries performed before 1997 were not registered automatically in the electronic charts at our hospital. Some data may have been lost in the process of digitalizing the paper charts.

\section{Conclusions}

Currently, more than 100,000 VNS devices have been implanted in more than 80,000 patients. VNS implantation is a relatively safe procedure but it still involves certain risks. The most common complications are postoperative hematoma, infection, and vocal cord paralysis. Although their occurrence rates are rather low at about $2 \%$, these complications may cause major suffering and even be life threatening. To reduce complications it is important to have a long-term perspective. The 25 years of follow-up of this study is of great strength considering that VNS can be a life-long treatment for many patients. Thus, it is important to include repeated surgeries, such as battery and lead replacements, given that complications also may occur with these surgeries. Children may have a greater risk for wound infection than adults due to behaviors more common in children. As concluded in the AAN guidelines, extra vigilance in monitoring for the occur- rence of site infection in children should be undertaken. Our general opinion is that VNS implantations should be restricted to neurosurgeons with detailed familiarity with the procedure at hand and attention to detail in order to minimize surgical complications.

\section{Acknowledgments}

This study was supported by Västra Götalandsregionen.

\section{References}

1. Air EL, Ghomri YM, Tyagi R, Grande AW, Crone K, Mangano FT: Management of vagal nerve stimulator infections: do they need to be removed? J Neurosurg Pediatr 3:73-78, 2009

2. Alexopoulos AV, Kotagal P, Loddenkemper T, Hammel J, Bingaman WE: Long-term results with vagus nerve stimulation in children with pharmacoresistant epilepsy. Seizure 15:491-503, 2006

3. Ali II, Pirzada NA, Kanjwal Y, Wannamaker B, Medhkour A, Koltz MT, et al: Complete heart block with ventricular asystole during left vagus nerve stimulation for epilepsy. Epilepsy Behav 5:768-771, 2004

4. Ardesch JJ, Buschman HP, van der Burgh PH, WagenerSchimmel LJ, van der Aa HE, Hageman G: Cardiac responses of vagus nerve stimulation: intraoperative bradycardia and subsequent chronic stimulation. Clin Neurol Neurosurg 109:849-852, 2007

5. Asconapé JJ, Moore DD, Zipes DP, Hartman LM, Duffell WH Jr: Bradycardia and asystole with the use of vagus nerve stimulation for the treatment of epilepsy: a rare complication of intraoperative device testing. Epilepsia 40:1452-1454, 1999

6. Ben-Menachem E: Vagus nerve stimulation, side effects, and long-term safety. J Clin Neurophysiol 18:415-418, 2001

7. Ben-Menachem E, Hellström K, Waldton C, Augustinsson LE: Evaluation of refractory epilepsy treated with vagus nerve stimulation for up to 5 years. Neurology 52:12651267, 1999

8. Ben-Menachem E, Mañon-Espaillat R, Ristanovic R, Wilder BJ, Stefan H, Mirza W, et al: Vagus nerve stimulation for treatment of partial seizures: 1 . A controlled study of effect on seizures. Epilepsia 35:616-626, 1994

9. Ben-Menachem E, Revesz D, Simon BJ, Silberstein S: Surgically implanted and non-invasive vagus nerve stimulation: a review of efficacy, safety and tolerability. Eur J Neurol 22:1260-1268, 2015

10. Ben-Menachem E, Rydenhag B, Silander H: Preliminary experience with a new system for vagus nerve stimulation for the treatment of refractory focal onset seizures. Epilepsy Behav 29:416-419, 2013

11. Coykendall DS, Gauderer MW, Blouin RR, Morales A: Vagus nerve stimulation for the management of seizures in children: an 8-year experience. J Pediatr Surg 45:1479-1483, 2010

12. Dlouhy BJ, Viljoen SV, Kung DK, Vogel TW, Granner MA, Howard MA III, et al: Vagus nerve stimulation after lead revision. Neurosurg Focus 32(3):E11, 2012

13. El Tahry R, Mollet L, Raedt R, Delbeke J, De Herdt V, Wyckhuys T, et al: Repeated assessment of larynx compound muscle action potentials using a self-sizing cuff electrode around the vagus nerve in experimental rats. J Neurosci Methods 198:287-293, 2011

14. Elliott RE, Morsi A, Kalhorn SP, Marcus J, Sellin J, Kang $\mathrm{M}$, et al: Vagus nerve stimulation in 436 consecutive patients with treatment-resistant epilepsy: long-term outcomes and predictors of response. Epilepsy Behav 20:57-63, 2011

15. Elliott RE, Rodgers SD, Bassani L, Morsi A, Geller EB, 
Carlson C, et al: Vagus nerve stimulation for children with treatment-resistant epilepsy: a consecutive series of 141 cases. J Neurosurg Pediatr 7:491-500, 2011

16. Granbichler CA, Nashef L, Selway R, Polkey CE: Mortality and SUDEP in epilepsy patients treated with vagus nerve stimulation. Epilepsia 56:291-296, 2015

17. Handforth A, DeGiorgio CM, Schachter SC, Uthman BM, Naritoku DK, Tecoma ES, et al: Vagus nerve stimulation therapy for partial-onset seizures: a randomized active-control trial. Neurology 51:48-55, 1998

18. Hornig GW, Murphy JV, Schallert G, Tilton C: Left vagus nerve stimulation in children with refractory epilepsy: an update. South Med J 90:484-488, 1997

19. Huf RL, Mamelak A, Kneedy-Cayem K: Vagus nerve stimulation therapy: 2-year prospective open-label study of 40 subjects with refractory epilepsy and low IQ who are living in long-term care facilities. Epilepsy Behav 6:417-423, 2005

20. Kabir SM, Rajaraman C, Rittey C, Zaki HS, Kemeny AA, McMullan J: Vagus nerve stimulation in children with intractable epilepsy: indications, complications and outcome Childs Nerv Syst 25:1097-1100, 2009

21. Kahlow H, Olivecrona M: Complications of vagal nerve stimulation for drug-resistant epilepsy: a single center longitudinal study of 143 patients. Seizure 22:827-833, 2013

22. Kang HC, Hwang YS, Kim DS, Kim HD: Vagus nerve stimulation in pediatric intractable epilepsy: a Korean bicentric study. Acta Neurochir Suppl 99:93-96, 2006

23. Kuba R, Brázdil M, Kalina M, Procházka T, Hovorka J, Nezádal T, et al: Vagus nerve stimulation: longitudinal followup of patients treated for 5 years. Seizure 18:269-274, 2009

24. Landy HJ, Ramsay RE, Slater J, Casiano RR, Morgan R: Vagus nerve stimulation for complex partial seizures: surgical technique, safety, and efficacy. J Neurosurg 78:26-31, 1993

25. Merrill CA, Jonsson MA, Minthon L, Ejnell H, Silander HC, Blennow $\mathrm{K}$, et al: Vagus nerve stimulation in patients with Alzheimer's disease: Additional follow-up results of a pilot study through 1 year. J Clin Psychiatry 67:1171-1178, 2006

26. Morris GL III, Gloss D, Buchhalter J, Mack KJ, Nickels K, Harden C: Evidence-based guideline update: vagus nerve stimulation for the treatment of epilepsy: report of the Guideline Development Subcommittee of the American Academy of Neurology. Epilepsy Curr 13:297-303, 2013

27. Murphy JV, Hornig GW, Schallert GS, Tilton CL: Adverse events in children receiving intermittent left vagal nerve stimulation. Pediatr Neurol 19:42-44, 1998

28. Neligan A, Bell GS, Shorvon SD, Sander JW: Temporal trends in the mortality of people with epilepsy: a review. Epilepsia 51:2241-2246, 2010

29. Ng WH, Donner E, Go C, Abou-Hamden A, Rutka JT: Revision of vagal nerve stimulation (VNS) electrodes: review and report on use of ultra-sharp monopolar tip. Childs Nerv Syst 26:1081-1084, 2010

30. Pastrana EA, Estronza S, Sosa IJ: Vagus nerve stimulation for intractable seizures in children: the University of Puerto Rico experience. P R Health Sci J 30:128-131, 2011

31. Patel NC, Edwards MS: Vagal nerve stimulator pocket infections. Pediatr Infect Dis J 23:681-683, 2004

32. Ramsay RE, Uthman BM, Augustinsson LE, Upton AR, Naritoku D, Willis J, et al: Vagus nerve stimulation for treatment of partial seizures: 2. Safety, side effects, and tolerability. Epilepsia 35:627-636, 1994

33. Robinson LC, Winston KR: Relationship of vocal cord paralysis to the coil diameter of vagus nerve stimulator leads. J Neurosurg 122:532-535, 2015
34. Rychlicki F, Zamponi N, Trignani R, Ricciuti RA, Iacoangeli M, Scerrati M: Vagus nerve stimulation: clinical experience in drug-resistant pediatric epileptic patients. Seizure 15:483490, 2006

35. Schuurman PR, Beukers RJ: Ventricular asystole during vagal nerve stimulation. Epilepsia 50:967-968, 2009

36. Sjögren MJ, Hellström PT, Jonsson MA, Runnerstam M, Silander HC, Ben-Menachem E: Cognition-enhancing effect of vagus nerve stimulation in patients with Alzheimer's disease: a pilot study. J Clin Psychiatry 63:972-980, 2002

37. Smyth MD, Tubbs RS, Bebin EM, Grabb PA, Blount JP: Complications of chronic vagus nerve stimulation for epilepsy in children. J Neurosurg 99:500-503, 2003

38. Spuck S, Tronnier V, Orosz I, Schönweiler R, Sepehrnia A, Nowak G, et al: Operative and technical complications of vagus nerve stimulator implantation. Neurosurgery 67 (2 Suppl Operative):489-494, 2010

39. Tanganelli P, Ferrero S, Colotto P, Regesta G: Vagus nerve stimulation for treatment of medically intractable seizures. Evaluation of long-term outcome. Clin Neurol Neurosurg 105:9-13, 2002

40. Tatum WO IV, Moore DB, Stecker MM, Baltuch GH, French JA, Ferreira JA, et al: Ventricular asystole during vagus nerve stimulation for epilepsy in humans. Neurology 52:12671269,1999

41. Vonck K, Thadani V, Gilbert K, Dedeurwaerdere S, De Groote L, De Herdt V, et al: Vagus nerve stimulation for refractory epilepsy: a transatlantic experience. J Clin Neurophysiol 21:283-289, 2004

42. Vonck K, Van Laere K, Dedeurwaerdere S, Caemaert J, De Reuck J, Boon P: The mechanism of action of vagus nerve stimulation for refractory epilepsy: the current status. J Clin Neurophysiol 18:394-401, 2001

43. Wheeler M, De Herdt V, Vonck K, Gilbert K, Manem S, Mackenzie T, et al: Efficacy of vagus nerve stimulation for refractory epilepsy among patient subgroups: a re-analysis using the Engel classification. Seizure 20:331-335, 2011

44. Zhang Y, Mazgalev TN: Arrhythmias and vagus nerve stimulation. Heart Fail Rev 16:147-161, 2011

\section{Disclosures}

The authors report no conflict of interest concerning the materials or methods used in this study or the findings specified in this paper.

\section{Author Contributions}

Conception and design: Révész, Rydenhag. Acquisition of data: Révész. Analysis and interpretation of data: Révész. Drafting the article: Révész, Rydenhag. Critically revising the article: Rydenhag, Ben-Menachem. Reviewed submitted version of manuscript: all authors. Approved the final version of the manuscript on behalf of all authors: Révész. Statistical analysis: Révész. Administrative/technical/material support: Révész. Study supervision: Rydenhag, Ben-Menachem.

\section{Correspondence}

David Révész, Institute of Neuroscience and Physiology, Department of Clinical Neuroscience and Rehabilitation, The Sahlgrenska Academy at the University of Gothenburg, Guldhedsgatan 19 , Göteborg SE-413 45, Sweden. email: david.revesz@neuro.gu.se. 